\title{
Local Existence for the Non-Resistive MHD Equations in Nearly Optimal Sobolev Spaces
}

\author{
Charles L. Fefferman, David S. McCormick, \\ JAMES C. RoBinson \& Jose L. RodRigo
}

Communicated by P. Constantin

\begin{abstract}
This paper establishes the local-in-time existence and uniqueness of solutions to the viscous, non-resistive magnetohydrodynamics (MHD) equations in $\mathbb{R}^{d}$, where $d=2,3$, with initial data $B_{0} \in H^{s}\left(\mathbb{R}^{d}\right)$ and $u_{0} \in H^{s-1+\varepsilon}\left(\mathbb{R}^{d}\right)$ for $s>d / 2$ and any $0<\varepsilon<1$. The proof relies on maximal regularity estimates for the Stokes equation. The obstruction to taking $\varepsilon=0$ is explained by the failure of solutions of the heat equation with initial data $u_{0} \in H^{s-1}$ to satisfy $u \in L^{1}\left(0, T ; H^{s+1}\right)$; we provide an explicit example of this phenomenon.
\end{abstract}

\section{Introduction}

In this paper we consider the equations of MHD with zero magnetic resistivity,

$$
\begin{aligned}
u_{t}-\Delta u+(u \cdot \nabla) u+\nabla p & =(B \cdot \nabla) B, & \nabla \cdot u & =0, \\
B_{t}+(u \cdot \nabla) B & =(B \cdot \nabla) u, & \nabla \cdot B & =0,
\end{aligned}
$$

along with specified initial data $u(0)=u_{0}$ and $B(0)=B_{0}$. Jiu and Niu [7] established the local existence of solutions in 2D for initial data in $H^{s}$ for integer $s \geqq 3$, and more recently REN et al. [12] and LIN et al. [10] have established the existence of global-in-time solutions for initial data sufficiently close to certain equilibrium solutions (again in 2D).

In a previous paper [6] we proved a local existence result for these equations taking arbitrary initial data in $u_{0}, B_{0} \in H^{s}\left(\mathbb{R}^{d}\right)$ with $s>d / 2$ for $d=2,3$. However, given the presence of the diffusive term in the equation for $u$, it is natural

DSMcC is supported by Leverhulme Trust research project grant RPG-2015-69. CLF is supported by NSF grant DMS-09-01040. JLR is supported by European Research Council grant 616797. 
to expect that such a local existence result should be possible with less regularity for $u_{0}$ than for $B_{0}$.

To this end, it was shown in [5] that one can prove local existence when the initial data $B_{0} \in B_{2,1}^{d / 2}\left(\mathbb{R}^{d}\right)$ and $u_{0} \in B_{2,1}^{d / 2-1}\left(\mathbb{R}^{d}\right)$. The underlying observation that allowed for such a result is that when $u_{0} \in B_{2,1}^{d / 2-1}\left(\mathbb{R}^{d}\right)$ the solution $u$ of the heat equation with initial data $u_{0}$ is an element of $L^{1}\left(0, T ; B_{2,1}^{d / 2+1}\left(\mathbb{R}^{d}\right)\right)$ : in these Besov spaces the solution regularises sufficiently that an additional two derivatives become integrable in time.

In Sobolev spaces this does not occur: in Lemma 2.1 we show that for $u_{0} \in H^{s}$ we have

$$
u \in L^{\infty}\left(0, T ; H^{s}\right) \cap L^{2}\left(0, T ; H^{s+1}\right) \cap L^{q}\left(0, T ; H^{s+2}\right)
$$

for any $0<q<1$, and this is in some sense the best possible. The failure of the estimate $u \in L^{1}\left(0, T ; H^{s+2}\right)$ is 'well known', but it is not easy to find any explicit example in the literature, so we provide one here in Lemma 2.2.

In this paper we therefore take $B_{0} \in H^{s}\left(\mathbb{R}^{d}\right)$, with $s>d / 2$, and $u_{0}$ slightly more regular than $H^{s-1}\left(\mathbb{R}^{d}\right)$, namely $u_{0} \in H^{s-1+\varepsilon}\left(\mathbb{R}^{d}\right)$, with $0<\varepsilon<1$. By making use of maximal regularity results for the heat equation (which we recall in the next section) we are then able to prove the local existence of a solution that remains bounded in these spaces (see Theorem 3.1 for a precise statement).

Throughout the paper we use the notation $\Lambda^{s}$ to denote the fractional derivative of order $s$, given in terms of the Fourier transform by

$$
\widehat{\Lambda^{s} f}=|\xi|^{s} \hat{f}
$$

We write

$$
\|u\|_{H^{s}}^{2}=\left\|\Lambda^{s} u\right\|^{2}+\|u\|^{2}, \quad s>0,
$$

which is equivalent to the standard $H^{s}$ norm when $s$ is a positive integer.

\section{Estimates for Solutions of the Heat Equation in Sobolev Spaces}

\subsection{Energy Estimates}

First we prove some standard estimates for solutions of the heat equation, including the $L^{q}\left(0, T ; H^{s+2}\right)$ estimate for $0<q<1$. We give the proofs, since we will need to keep careful track of the dependence of the estimates on $T$; for simplicity we restrict to $T \leqq 1$, which will of course be sufficient for local existence arguments.

We use relatively elementary energy methods, but the results can be derived by other means; for example, the fact that $u \in L^{q}\left(0, T ; H^{s+2}\right)$ follows from the smoothing estimate $\left\|\partial^{\beta} \mathrm{e}^{\Delta t} f\right\|_{L^{2}} \leqq C t^{-|\beta| / 2}\|f\|_{L^{2}}$, as kindly pointed out by the referee. 
Lemma 2.1. If $u_{0} \in H^{s}\left(\mathbb{R}^{d}\right)$ and $u$ denotes the solution of the heat equation

$$
\partial_{t} u=\Delta u \quad \text { with } \quad u(0)=u_{0}
$$

then $u \in L^{\infty}\left(0, T ; H^{s}\right) \cap L^{2}\left(0, T ; H^{s+1}\right)$ and $\sqrt{t} u \in L^{2}\left(0, T ; H^{s+2}\left(\mathbb{R}^{d}\right)\right)$. When $T \leqq 1$

$$
\sup _{0 \leqq t \leqq T}\|u(t)\|_{H^{s}}^{2}, \quad \int_{0}^{T}\|u(t)\|_{H^{s+1}}^{2}, \quad \int_{0}^{T} t\|u(t)\|_{H^{s+2}}^{2} \leqq\left\|u_{0}\right\|_{H^{s}}^{2} .
$$

Consequently $u \in L^{q}\left(0, T ; H^{s+2}\left(\mathbb{R}^{d}\right)\right)$ for any $0<q<1$, and

$$
\int_{0}^{T}\|u(t)\|_{H^{s+2}}^{q} \mathrm{~d} s \leqq C_{q} T^{1-q}\left\|u_{0}\right\|_{H^{s}}^{q}
$$

provided that $T \leqq 1$.

Proof. We start with the $L^{2}$ estimate obtained by taking the inner product with $u$ in $L^{2}$ to give

$$
\frac{1}{2} \frac{\mathrm{d}}{\mathrm{d} t}\|u\|^{2}+\|\nabla u\|^{2}=0
$$

and then integrating in time,

$$
\frac{1}{2}\|u(t)\|^{2}+\int_{0}^{t}\|\nabla u(\tau)\|^{2} \mathrm{~d} \tau=\frac{1}{2}\left\|u_{0}\right\|^{2} .
$$

To bound the higher derivatives we act on the equations with $\Lambda^{s}$ and then take the $L^{2}$ inner product with $\Lambda^{s} u$ to obtain

$$
\frac{1}{2} \frac{\mathrm{d}}{\mathrm{d} t}\left\|\Lambda^{s} u\right\|^{2}+\left\|\Lambda^{s+1} u\right\|^{2}=0
$$

followed by an integration in time,

$$
\frac{1}{2}\left\|\Lambda^{s} u(t)\right\|^{2}+\int_{0}^{t}\left\|\Lambda^{s+1} u(\tau)\right\|^{2} \mathrm{~d} \tau=\frac{1}{2}\left\|\Lambda^{s} u_{0}\right\|^{2} .
$$

Combining these two estimates shows that $u \in L^{\infty}\left(0, T ; H^{s}\right) \cap L^{2}\left(0, T ; H^{s+1}\right)$, with

$$
\sup _{0 \leq t \leq T}\|u(t)\|_{H^{s}}^{2} \leqq\left\|u_{0}\right\|_{H^{s}}^{2}
$$

and

$$
\int_{0}^{T}\|u\|_{H^{s+1}}^{2} \leqq \frac{1}{2}\left\|\Lambda^{s} u_{0}\right\|^{2}+T\left\|u_{0}\right\|^{2}
$$

in particular, if $T \leqq 1$, then

$$
\int_{0}^{T}\|u\|_{H^{s+1}}^{2} \leqq\left\|u_{0}\right\|_{H^{s}}^{2} .
$$


To obtain the bound on $\sqrt{t} u$ in $H^{s+2}$ we act on the equations with $\Lambda^{s+1}$ and take the $L^{2}$ inner product with $t \Lambda^{s+1} u$. Then

$$
\frac{1}{2} \frac{\mathrm{d}}{\mathrm{d} t}\left(t\left\|\Lambda^{s+1} u\right\|^{2}\right)-\frac{1}{2}\left\|\Lambda^{s+1} u\right\|^{2}+t\left\|\Lambda^{s+2} u\right\|^{2}=0 .
$$

Integrating from 0 to $T$ yields

$$
\frac{T}{2}\left\|\Lambda^{s+1} u(T)\right\|^{2}+\int_{0}^{T} t\left\|\Lambda^{s+2} u(t)\right\|^{2} \mathrm{~d} t \leqq \frac{1}{2} \int_{0}^{T}\left\|\Lambda^{s+1} u(t)\right\|^{2} \mathrm{~d} t \leqq \frac{1}{4}\left\|\Lambda^{s} u_{0}\right\|^{2},
$$

using the bound from (2.2). We also have

$$
\int_{0}^{T} t\|u(t)\|^{2} \mathrm{~d} t \leqq \frac{T^{2}}{2}\left\|u_{0}\right\|^{2}
$$

from which it follows that $\sqrt{t} u \in L^{2}\left(0, T ; H^{s+2}\right)$. For $T \leqq 1$ we can combine this with (2.3) to obtain the estimate

$$
\int_{0}^{T} t\|u\|_{H^{s+2}}^{2} \mathrm{~d} t \leqq \frac{1}{2}\left\|u_{0}\right\|_{H^{s}}^{2} .
$$

For any $q<1$ we have, using Hölder's inequality with exponents $2 / q$ and $2 /(2-q)$,

$$
\begin{aligned}
\int_{0}^{T}\|u(t)\|_{H^{s+2}}^{q} & =\int_{0}^{T} t^{-q / 2} t^{q / 2}\|u(t)\|_{H^{s+2}}^{q} \\
& \leqq\left(\int_{0}^{T} t^{-q /(2-q)}\right)^{1-(q / 2)}\left(\int_{0}^{T} t\|u(t)\|_{H^{s+2}}^{2}\right)^{q / 2} \\
& \leqq C_{q} T^{1-q}\left\|u_{0}\right\|_{H^{s}}^{q},
\end{aligned}
$$

since $0<q<1$ ensures that $q /(2-q)<1$ and the first term is integrable.

\subsection{An Example of $u_{0} \in L^{2}$ with $u \notin L^{1}\left(0, T ; H^{2}\right)$}

We now provide an explicit example of an initial condition $u_{0} \in L^{2}\left(\mathbb{R}^{d}\right)$ such that $u \notin L^{1}\left(0, T ; H^{2}\left(\mathbb{R}^{d}\right)\right)$.

Lemma 2.2. There exists $u_{0} \in L^{2}\left(\mathbb{R}^{d}\right)$ such that the solution $u$ of the heat equation with initial data $u_{0}$ is not an element of $L^{1}\left(0, T ; H^{2}\left(\mathbb{R}^{d}\right)\right)$.

Proof. We let $u_{0}$ be the function with Fourier transform

$$
\hat{u}_{0}(\xi)=\frac{1}{|\xi|^{d / 2} \log (2+|\xi|)} .
$$

The solution $u(t)$ of the heat equation with initial data $u_{0}$ has Fourier transform

$$
\hat{u}(t, \xi)=\hat{u}_{0}(\xi) \mathrm{e}^{-|\xi|^{2} t}
$$


and

$$
\|u(t)\|_{\dot{H}^{2}}^{2}=\int_{\mathbb{R}^{d}}|\xi|^{4}\left|\hat{u}_{0}(\xi)\right|^{2} \mathrm{e}^{-2|\xi|^{2} t} \mathrm{~d} \xi
$$

We therefore have

$$
I:=\int_{0}^{T}\|u(t)\|_{\dot{H}^{2}} \mathrm{~d} t=\int_{0}^{T}\left(\int_{\mathbb{R}^{d}}|\xi|^{4}\left|\hat{u}_{0}(\xi)\right|^{2} \mathrm{e}^{-2|\xi|^{2} t} \mathrm{~d} \xi\right)^{1 / 2} \mathrm{~d} t .
$$

In order to bound this from below we split the range of time integration, choosing $j_{0}$ such that $j_{0}^{-2} \leqq T$, and write

$$
\begin{aligned}
I & \geqq \sum_{j=j_{0}}^{N} \int_{(j+1)^{-2}}^{j^{-2}}\left(\int_{\mathbb{R}^{d}}|\xi|^{4}\left|\hat{u}_{0}(\xi)\right|^{2} \mathrm{e}^{-2|\xi|^{2} t} \mathrm{~d} \xi\right)^{1 / 2} \mathrm{~d} t \\
& \geqq \sum_{j=j_{0}}^{N} \int_{(j+1)^{-2}}^{j^{-2}}\left(\int_{|\xi| \leqq j}|\xi|^{4}\left|\hat{u}_{0}(\xi)\right|^{2} \mathrm{e}^{-2|\xi|^{2} t} \mathrm{~d} \xi\right)^{1 / 2} \mathrm{~d} t \\
& \geqq \mathrm{e}^{-1} \sum_{j=j_{0}}^{N} \int_{(j+1)^{-2}}^{j^{-2}}\left(\int_{|\xi| \leqq j}|\xi|^{4}\left|\hat{u}_{0}(\xi)\right|^{2} \mathrm{~d} \xi\right)^{1 / 2} \mathrm{~d} t \\
& \geqq \mathrm{e}^{-1} \sum_{j=j_{0}}^{N} \frac{1}{j^{2}(j+1)}\left(\int_{|\xi| \leqq j}|\xi|^{4}\left|\hat{u}_{0}(\xi)\right|^{2} \mathrm{~d} \xi\right)^{1 / 2} .
\end{aligned}
$$

By our choice of $\hat{u}_{0}(\xi)$ in (2.4) we have

$$
\begin{aligned}
\int_{|\xi| \leqq j}|\xi|^{4}\left|\hat{u}_{0}(\xi)\right|^{2} \mathrm{~d} \xi & =\int_{|\xi| \leqq j}|\xi|^{4} \frac{1}{|\xi|^{d}[\log (2+|\xi|)]^{2}} \mathrm{~d} \xi \\
& \geqq \frac{1}{[\log (2+j)]^{2}} \int_{|\xi| \leqq j}|\xi|^{4-d} \mathrm{~d} \xi \\
& \geqq \frac{c}{[\log (2+j)]^{2}} j^{4}
\end{aligned}
$$

It follows that

$$
I \geqq c \mathrm{e}^{-1} \sum_{j=j_{0}}^{N} \frac{1}{(j+1) \log (2+j)},
$$

as since the sum is unbounded as $N \rightarrow \infty$ it follows that $u \notin L^{1}\left(0, T ; \dot{H}^{2}\right)$ as claimed. 


\subsection{Maximal Regularity-Type Results}

Usually, 'maximal regularity' results for the heat equation yield

$$
\partial_{t} u, \quad \Delta u \in L^{p}\left(0, T ; L^{q}\right)
$$

when $u$ solves

$$
\partial_{t} u-\Delta u=f, \quad u(0)=0
$$

with $f \in L^{p}\left(0, T ; L^{q}\right)$. The results follow from maximal regularity when $p=q$, obtained as inequality (3.1) in Chapter IV, Section 3, pages 289-290 of [9] using the Mihlin Multiplier Theorem [11] and then an interpolation theorem due to BENEDEK et al. [1]; the procedure is clearly explained in [8], for example. However, in this paper we will only require the following $L^{2}$-based Sobolev-space result, for which the basic $L^{2}\left(0, T ; L^{2}\right)$ maximal regularity estimate can be obtained relatively easily.

Proposition 2.3. There exists a constant $C_{r}$ such that if $f \in L^{r}\left(0, T ; H^{s}\right), s \geqq 0$, and $u$ satisfies

$$
\partial_{t} u-\Delta u=f, \quad u(0)=0,
$$

then $u \in L^{r}\left(0, T ; \dot{H}^{s+2}\right)$ with

$$
\|u\|_{L^{r}\left(0, T ; \dot{H}^{s+2}\right)} \leqq C_{r}\|f\|_{L^{r}\left(0, T ; H^{s}\right)} .
$$

The constant $C_{r}$ can be chosen uniformly for all $0 \leqq T \leqq 1$.

Proof. First we treat the case $s=0$, that is we bound $u \in L^{r}\left(0, T ; H^{2}\right)$ in terms of $f \in L^{r}\left(0, T ; L^{2}\right)$. The passage from estimates for the case $r=2$ to the case $1<r<\infty$ is covered in [8]. We therefore only prove the estimates in the case $r=2$.

The $L^{2}$ norm of $u$ is bounded simply by taking the $L^{2}$ inner product with $u$, using the Cauchy-Schwarz inequality, and integrating in time:

$$
\frac{1}{2} \frac{\mathrm{d}}{\mathrm{d} t}\|u\|^{2}+\|\nabla u\|^{2}=\langle f, u\rangle \leqq\|f\|\|u\| \leqq \frac{1}{2}\|u\|^{2}+\frac{1}{2}\|f\|^{2},
$$

and so

$$
\frac{\mathrm{d}}{\mathrm{d} t}\left[\mathrm{e}^{-t}\|u\|^{2}\right] \leqq \mathrm{e}^{-t}\|f(t)\|^{2}
$$

which implies (since $u(0)=0$ ) that

$$
\|u(t)\|^{2} \leqq \int_{0}^{t}\|f(s)\|^{2} \mathrm{e}^{t-s} \mathrm{~d} s,
$$

whence

$$
\begin{aligned}
\int_{0}^{T}\|u(t)\|^{2} & \leqq \int_{0}^{T} \int_{0}^{t}\|f(s)\|^{2} \mathrm{e}^{t-s} \mathrm{~d} s \mathrm{~d} t \\
& =\int_{0}^{T} \int_{s}^{T}\|f(s)\|^{2} \mathrm{e}^{t-s} \mathrm{~d} t \mathrm{~d} s \leqq \mathrm{e}^{T} \int_{0}^{T}\|f(s)\|^{2} \mathrm{~d} s,
\end{aligned}
$$


that is

$$
\|u\|_{L^{2}\left(0, T ; L^{2}\right)} \leqq \mathrm{e}^{T}\|f\|_{L^{2}\left(0, T ; L^{2}\right)} .
$$

For $\|u\|_{\dot{H}^{2}}$ we can argue directly from the Fourier transform of the solution $u$, since

$$
\widehat{\Lambda^{2} u}(\xi, t)=\int_{0}^{t}|\xi|^{2} \mathrm{e}^{-|\xi|^{2}(t-s)} \hat{f}(s, \xi) \mathrm{d} s
$$

Define

$$
G(\xi, t)= \begin{cases}|\xi|^{2} \mathrm{e}^{-|\xi|^{2} t} & t \geqq 0 \\ 0 & t<0\end{cases}
$$

and

$$
F(\xi, t)= \begin{cases}\hat{f}(\xi ; t) & 0 \leqq t \leqq T \\ 0 & \text { otherwise }\end{cases}
$$

Then

$$
\widehat{\Lambda^{2} u}(\xi ; t)=\int_{\mathbb{R}} G(\xi, t-s) F(\xi, s) \mathrm{d} s
$$

and we can use Young's inequality for convolutions to give

$$
\left\|\widehat{\Lambda^{2} u}(\xi, \cdot)\right\|_{L^{2}(0, T)} \leqq\left\|\widehat{\Lambda^{2} u}(\xi, \cdot)\right\|_{L^{2}(\mathbb{R})} \leqq\|F(\xi, \cdot)\|_{L^{2}(\mathbb{R})}=\|\hat{f}(\xi, \cdot)\|_{L^{2}(0, T)},
$$

since $\|G(\xi, \cdot)\|_{L^{1}}=1$. Therefore

$$
\|u\|_{L^{2}\left(0, T ; \dot{H}^{2}\right)}=\left\|\widehat{\Lambda^{2} u}\right\|_{L^{2}\left((0, T) \times \mathbb{R}^{d}\right)} \leqq\|\hat{f}\|_{L^{2}\left((0, T) \times \mathbb{R}^{d}\right)}=\|f\|_{L^{2}\left(0, T ; L^{2}\right)} .
$$

Combined with (2.6) this yields $\|u\|_{L^{2}\left(0, T ; \dot{H}^{2}\right)} \leqq C_{2}\|f\|_{L^{2}\left(0, T ; L^{2}\right)}$, and hence (via the results of [1]) we obtain

$$
\|u\|_{L^{r}\left(0, T ; \dot{H}^{2}\right)} \leqq C_{r}\|f\|_{L^{r}\left(0, T ; L^{2}\right)} .
$$

Letting $C_{r}$ be the constant for the choice $T=1$, it can easily be seen that this constant is also valid for $T \leqq 1$ by extending $f$ to be zero on the interval $(T, 1]$.

We now apply this estimate to $u=v$ and $u=\Lambda^{s} v$ : we obtain

$$
\|v\|_{L^{r}\left(0, T ; \dot{H}^{2}\right)} \leqq C_{r}\|f\|_{L^{r}\left(0, T ; L^{2}\right)}
$$

and

$\|v\|_{L^{r}\left(0, T ; \dot{H}^{s+2}\right)}=\left\|\Lambda^{s} v\right\|_{L^{r}\left(0, T ; \dot{H}^{2}\right)} \leqq C_{r}\left\|\Lambda^{s} f\right\|_{L^{r}\left(0, T ; L^{2}\right)}=C_{r}\|f\|_{L^{r}\left(0, T ; \dot{H}^{s}\right)}$, from which (2.5) follows. 
We will apply this result in combination with the regularity results for the heat equation from Lemma 2.1 in the following form for solutions of the Stokes equation, allowing for non-zero initial data. Note that in order to obtain an $L^{1}$-in-time estimate on $\|u\|_{H^{s+1}}$ we require $L^{r}$ integrability of $f$ with $r>1$, and the initial data to be in $H^{s-1+\varepsilon}$. [Considering the equations in Besov spaces as in [5] allows for $r=1$ and $\varepsilon=0$ (and $s=d / 2$ rather than $s>d / 2$ in the final results)].

Corollary 2.4. If $f \in L^{r}\left(0, T ; H^{s-1}\right), 1<r<\infty, s>1$, and

$$
\partial_{t} u-\Delta u+\nabla p=f, \quad \nabla \cdot u=0, \quad u(0)=u_{0} \in H^{s-1+\varepsilon},
$$

where $u_{0}$ is divergence free, then for $T \leqq 1$

$$
\int_{0}^{T}\|u\|_{H^{s+1}} \leqq C_{\varepsilon} T^{\varepsilon / 2}\left\|u_{0}\right\|_{H^{s-1+\varepsilon}}+C_{r} T^{1-\frac{1}{r}}\|f\|_{L^{r}\left(0, T ; H^{s-1}\right)} .
$$

Proof. First we consider the solution $v$ of

$$
\partial_{t} v-\Delta v+\nabla \pi=0, \quad \nabla \cdot v=0, \quad v(0)=u_{0} .
$$

Since $u_{0}$ is divergence free, if we apply the Leray projector $\mathbb{P}$ (orthogonal projection onto elements of $L^{2}$ whose weak divergence is zero) we obtain

$$
\partial_{t} v-\Delta v=0, \quad v(0)=u_{0},
$$

since $\mathbb{P}$ commutes with derivatives on the whole space. It follows that $v$ is in fact the solution of the heat equation with initial data $u_{0}$. We can therefore use Lemma 2.1 to ensure that

$$
v \in L^{\infty}\left(0, T ; H^{s-1+\varepsilon}\right) \cap L^{2}\left(0, T ; H^{s+\varepsilon}\right) \cap L^{q}\left(0, T ; H^{s+\varepsilon+1}\right)
$$

for any $q<1$, with all these norms depending only on the norm of the initial data in $H^{s-1+\varepsilon}$. It follows by interpolation that $v \in L^{1}\left(0, T ; H^{s+1}\right)$ with

$$
\begin{aligned}
\int_{0}^{T}\|v\|_{H^{s+1}} & \leqq \int_{0}^{T}\|v\|_{H^{s+1+\varepsilon}}^{1-\varepsilon}\|v\|_{H^{s+\varepsilon}}^{\varepsilon} \\
& \leqq\left(\int_{0}^{T}\|v\|_{H^{s+1+\varepsilon}}^{2(1-\varepsilon) /(2-\varepsilon)}\right)^{(2-\varepsilon) / 2}\left(\int_{0}^{T}\|v\|_{H^{s+\varepsilon}}^{2}\right)^{\varepsilon / 2},
\end{aligned}
$$

using Hölder's inequality with exponents $(2 /(2-\varepsilon), 2 / \varepsilon)$. Noting that

$$
\frac{2(1-\varepsilon)}{2-\varepsilon}=1-\frac{\varepsilon}{2-\varepsilon}<1
$$

we can use Lemma 2.1 to obtain

$$
\int_{0}^{T}\|v\|_{H^{s+1}} \leqq C_{\varepsilon} T^{\varepsilon / 2}\left\|u_{0}\right\|_{H^{s-1+\varepsilon}}
$$

The difference $w=u-v$ satisfies

$$
\partial_{t} w-\Delta w+\nabla \theta=f, \quad \nabla \cdot w=0, \quad w(0)=0 .
$$


Again we can apply the Leray projector $\mathbb{P}$, which is bounded from $H^{s-1}$ into $H^{s-1}$, to obtain

$$
\partial_{t} w-\Delta w=\mathbb{P} f, \quad w(0)=0 .
$$

By the maximal regularity results for the heat equation from Proposition 2.3, we know that for any $r>1$ we have

$$
\begin{aligned}
\int_{0}^{T}\|w\|_{H^{s+1}} & \leqq T^{1 / r^{\prime}}\|w\|_{L^{r}\left(0, T ; H^{s+1}\right)} \\
& \leqq C_{r} T^{1 / r^{\prime}}\|\mathbb{P} f\|_{L^{r}\left(0, T ; H^{s-1}\right)} \\
& \leqq C_{r} T^{1 / r^{\prime}}\|f\|_{L^{r}\left(0, T ; H^{s-1}\right)}
\end{aligned}
$$

where $\left(r, r^{\prime}\right)$ are conjugate. The inequality in (2.7) now follows by combining (2.8) and (2.9).

\section{Proof of Local Existence}

The main part of the proof consists of a priori estimates, which we prove formally. To make the proof rigorous requires some approximation procedure such as that employed in [6], to which we refer for the details. Where the limiting process involved would turns equalities into inequalities, we write inequalities even in these formal estimates.

Theorem 3.1. Let $d=2,3$. Take $s>d / 2$ and $0<\varepsilon<1$. Suppose that the initial conditions satisfy $B_{0} \in H^{s}\left(\mathbb{R}^{d}\right)$ and $u_{0} \in H^{s-1+\varepsilon}\left(\mathbb{R}^{d}\right)$. Then there exists $T_{*}>0$ such that the non-resistive MHD system (1.1) has a solution $(u, B)$ with

$$
u \in L^{\infty}\left(0, T_{*} ; H^{s-1+\varepsilon}\left(\mathbb{R}^{d}\right)\right) \cap L^{2}\left(0, T_{*} ; H^{s+\varepsilon}\left(\mathbb{R}^{d}\right)\right) \cap L^{1}\left(0, T_{*} ; H^{s+1}\left(\mathbb{R}^{d}\right)\right)
$$

and

$$
B \in L^{\infty}\left(0, T_{*} ; H^{s}\left(\mathbb{R}^{d}\right)\right)
$$

Note that the case $\varepsilon=1$ was covered in a previous paper [6] and is specifically excluded here.

Proof. Throughout the proof various constants will depend on $s$, but we do not track this dependency.

We first obtain a basic energy estimate in $L^{2}$. We take the $L^{2}$ inner product of the $u$ equation with $u$ and of the $B$ equation with $B$ to obtain

$$
\begin{aligned}
\frac{1}{2} \frac{\mathrm{d}}{\mathrm{d} t}\|u\|^{2}+\|\nabla u\|^{2} & =\langle(B \cdot \nabla) B, u\rangle \quad \text { and } \\
\frac{1}{2} \frac{\mathrm{d}}{\mathrm{d} t}\|B\|^{2} & =\langle(B \cdot \nabla) u, B\rangle .
\end{aligned}
$$


Since $\langle(B \cdot \nabla) u, B\rangle=-\langle(B \cdot \nabla) u, B\rangle$ we can add the two equations to yield

$$
\frac{1}{2} \frac{\mathrm{d}}{\mathrm{d} t}\left(\|u\|^{2}+\|B\|^{2}\right)+\|\nabla u\|^{2} \leqq 0
$$

and so

$$
\|u(t)\|^{2}+\|B(t)\|^{2}+2 \int_{0}^{t}\|\nabla u(s)\|^{2} \mathrm{~d} s \leqq\left\|u_{0}\right\|^{2}+\left\|B_{0}\right\|^{2}=: M_{0} .
$$

It is also helpful to have two other estimates for later use; observing that

$$
|\langle(B \cdot \nabla) u, B\rangle| \leqq c\|B\|\|\nabla u\|\|B\|_{L^{\infty}}
$$

and using the embedding $H^{s} \subset L^{\infty}$ (valid since $s>d / 2$ ) and Young's inequality we obtain

$$
\frac{\mathrm{d}}{\mathrm{d} t}\|u\|^{2}+\|\nabla u\|^{2} \leqq c\|B\|^{2}\|B\|_{H^{s}}^{2} \leqq c\|B\|_{H^{s}}^{4} ;
$$

and similarly, since $|\langle(B \cdot \nabla) u, B\rangle| \leqq\|B\|^{2}\|\nabla u\|_{L^{\infty}}$,

$$
\frac{1}{2} \frac{\mathrm{d}}{\mathrm{d} t}\|B\|^{2} \leqq c\|\nabla u\|_{H^{s}}\|B\|^{2} .
$$

In order to estimate the norm of $B$ in $H^{s}$ we act on the $B$ equation with $\Lambda^{s}$ and take the inner product with $\Lambda^{s} B$ in $L^{2}$. This yields

$$
\begin{aligned}
\frac{1}{2} \frac{\mathrm{d}}{\mathrm{d} t}\left\|\Lambda^{s} B\right\|^{2} & \leqq\left|\left\langle\Lambda^{s}[(B \cdot \nabla) u], \Lambda^{s} B\right\rangle\right|+\left|\left\langle\Lambda^{s}[(u \cdot \nabla) B], \Lambda^{s} B\right\rangle\right| \\
& \leqq c\|\nabla u\|_{H^{s}}\|B\|_{H^{s}}^{2},
\end{aligned}
$$

using the fact that $H^{s}$ is an algebra since $s>d / 2$, along with the estimate proved in [6], that

$$
\left|\left\langle\Lambda^{s}[(u \cdot \nabla) B], \Lambda^{s} B\right\rangle\right| \leqq c\|\nabla u\|_{H^{s}}\|B\|_{H^{s}}^{2},
$$

valid when $s>d / 2$. Combined with (3.3) this yields

$$
\frac{1}{2} \frac{\mathrm{d}}{\mathrm{d} t}\|B\|_{H^{s}}^{2} \leqq c\|\nabla u\|_{H^{s}}\|B\|_{H^{s}}^{2}
$$

The estimates for the $u$ equation are more delicate. First we obtain estimates on $u$ in the space $L^{1}\left(0, T ; H^{s+1}\right)$, using the maximal regularity estimates from Proposition 2.3 and Corollary 2.4. We consider the equation for $u$ as the forced Stokes equation

$$
\partial_{t} u-\Delta u+\nabla p=f:=-(u \cdot \nabla) u+(B \cdot \nabla) B, \quad \nabla \cdot u=0, \quad u(0)=u_{0},
$$

and so estimate (2.7) from Corollary 2.4 yields

$$
\int_{0}^{T}\|u\|_{H^{s+1}} \leqq C_{\varepsilon} T^{\varepsilon / 2}\left\|u_{0}\right\|_{H^{s-1+\varepsilon}}+C_{r} T^{1-\frac{1}{r}}\|f\|_{L^{r}\left(0, T ; H^{s-1}\right)} .
$$


Since

$$
\begin{aligned}
\|f\|_{H^{s-1}} & =\|\nabla \cdot(B \otimes B)-\nabla \cdot(u \otimes u)\|_{H^{s-1}} \\
& \leqq\|B \otimes B\|_{H^{s}}+\|u \otimes u\|_{H^{s}} \\
& \leqq c\|B\|_{H^{s}}^{2}+c\|u\|_{H^{s}}^{2} \\
& \leqq c\|B\|_{H^{s}}^{2}+c\|u\|^{2 \varepsilon /(s+\varepsilon)}\|u\|_{H^{s+\varepsilon}}^{2 s /(s+\varepsilon)} \\
& \leqq\|B\|_{H^{s}}^{2}+c M_{0}^{\varepsilon /(s+\varepsilon)}\|u\|_{H^{s+\varepsilon}}^{2 s /(s+\varepsilon)},
\end{aligned}
$$

using (3.1), if we choose $r=(s+\varepsilon) / s>1$ then from (3.6) we have

$$
\begin{aligned}
\int_{0}^{T}\|u\|_{H^{s+1}} \leqq & C_{\varepsilon} T^{\varepsilon / 2}\left\|u_{0}\right\|_{H^{s-1+\varepsilon}} \\
& +C_{\varepsilon} T^{\varepsilon /(s+\varepsilon)}\left(\int_{0}^{T}\|B\|_{H^{s}}^{2(s+\varepsilon) / s}+c M_{0}^{\varepsilon / s}\|u\|_{H^{s+\varepsilon}}^{2} \mathrm{~d} \tau\right)^{s /(s+\varepsilon)} .
\end{aligned}
$$

We now estimate the norm of $u$ in $H^{s-1+\varepsilon}$ and $H^{s+\varepsilon}$. If we act with $\Lambda^{s-1+\varepsilon}$ on the $u$ equation and take the inner product with $\Lambda^{s-1+\varepsilon} u$, then

$$
\begin{aligned}
\frac{1}{2} \frac{\mathrm{d}}{\mathrm{d} t} \| & \Lambda^{s-1+\varepsilon} u\left\|^{2}+\right\| \Lambda^{s+\varepsilon} u \|^{2} \\
& \leqq-\left\langle\Lambda^{s-1+\varepsilon}[(u \cdot \nabla) u], \Lambda^{s-1+\varepsilon} u\right\rangle+\left\langle\Lambda^{s-1+\varepsilon}[(B \cdot \nabla) B], \Lambda^{s-1+\varepsilon} u\right\rangle .
\end{aligned}
$$

For the first term on the right-hand side, we write

$$
\begin{aligned}
\left.\| \Lambda^{s-1+\varepsilon}[(u \cdot \nabla) u], \Lambda^{s-1+\varepsilon} u\right\rangle & =\left|\left\langle\Lambda^{s-1}[(u \cdot \nabla) u], \Lambda^{s-1+2 \varepsilon} u\right\rangle\right| \\
& =\|\left\langle\Lambda^{s-1}[\nabla \cdot(u \otimes u)], \Lambda^{s-1+2 \varepsilon} u\right\rangle \mid \\
& \leqq c\|u\|_{H^{s}}^{2}\|u\|_{H^{s-1+2 \varepsilon}} \\
& \leqq c\left(\|u\|_{H^{s-1+\varepsilon}}^{\varepsilon}\|u\|_{H^{s+\varepsilon}}^{1-\varepsilon}\right)^{2}\|u\|_{H^{s-1+\varepsilon}}^{1-\varepsilon}\|u\|_{H^{s+\varepsilon}}^{\varepsilon} \\
& \leqq c\|u\|_{H^{s-1+\varepsilon}}^{1+\varepsilon}\|u\|_{H^{s+\varepsilon}}^{2-\varepsilon} \\
& \leqq c\|u\|_{H^{s-1+\varepsilon}}^{2(1+\varepsilon) / \varepsilon}+\frac{1}{4}\|u\|_{H^{s+\varepsilon}}^{2},
\end{aligned}
$$

where we have used Sobolev interpolation, Young's inequality, and the fact that $H^{s}$ is an algebra (as $s>d / 2$ ). The second term is handled similarly:

$$
\begin{aligned}
\left|\left\langle\Lambda^{s-1+\varepsilon}[(B \cdot \nabla) B], \Lambda^{s-1+\varepsilon} u\right\rangle\right| & =\left|\left\langle\Lambda^{s-1}[(B \cdot \nabla) B], \Lambda^{s-1+2 \varepsilon} u\right\rangle\right| \\
& =\left|\left\langle\Lambda^{s-1}[\nabla \cdot(B \otimes B)], \Lambda^{s-1+2 \varepsilon} u\right\rangle\right| \\
& \leqq c\|B\|_{H^{s}}^{2}\|u\|_{H^{s-1+2 \varepsilon}} \\
& \leqq c\|B\|_{H^{s}}^{2}\|u\|_{H^{s-1+\varepsilon}}^{1-\varepsilon}\|u\|_{H^{s+\varepsilon}}^{\varepsilon} \\
& \leqq c\|B\|_{H^{s}}^{2(1+\varepsilon)}+c\|u\|_{H^{s-1+\varepsilon}}^{2(1+\varepsilon) / \varepsilon}+\frac{1}{4}\|u\|_{H^{s+\varepsilon}}^{2},
\end{aligned}
$$


using the three-term Young's inequality with exponents $\left(1+\varepsilon, \frac{2(1+\varepsilon)}{\varepsilon(1-\varepsilon)}, \frac{2}{\varepsilon}\right)$. Combining these yields ${ }^{1}$

$$
\frac{1}{2} \frac{\mathrm{d}}{\mathrm{d} t}\left\|\Lambda^{s-1+\varepsilon} u\right\|^{2}+\left\|\Lambda^{s+\varepsilon} u\right\|^{2} \leqq c\|B\|_{H^{s}}^{2(1+\varepsilon)}+c\|u\|_{H^{s-1+\varepsilon}}^{2(1+\varepsilon) / \varepsilon}+\frac{1}{2}\|u\|_{H^{s+\varepsilon}}^{2} \cdot 1
$$

If we add (3.2) and an additional term $+\|u\|^{2}$ to both sides then we obtain

$$
\begin{aligned}
& \frac{1}{2} \frac{\mathrm{d}}{\mathrm{d} t}\|u\|_{H^{s-1+\varepsilon}}^{2}+\|u\|_{H^{s+\varepsilon}}^{2} \\
& \quad \leqq c\|u\|_{H^{s-1+\varepsilon}}^{2(1+\varepsilon) / \varepsilon}+c\|B\|_{H^{s}}^{2(1+\varepsilon)}+c\|B\|_{H^{s}}^{4}+\frac{1}{2}\|u\|_{H^{s+\varepsilon}}^{2}+\|u\|^{2},
\end{aligned}
$$

and so

$$
\frac{\mathrm{d}}{\mathrm{d} t}\|u\|_{H^{s-1+\varepsilon}}^{2}+\|u\|_{H^{s+\varepsilon}}^{2} \leqq c_{1}\|u\|_{H^{s-1+\varepsilon}}^{2(1+\varepsilon) / \varepsilon}+c_{2}\|B\|_{H^{s}}^{2(1+\varepsilon)}+c_{3}\|B\|_{H^{s}}^{4}+2\|u\|^{2} .
$$

We now have three ingredients: the differential inequality (3.9) for $u$; the $B$ equation (3.5)

$$
\frac{1}{2} \frac{\mathrm{d}}{\mathrm{d} t}\|B\|_{H^{s}}^{2} \leqq c_{4}\|\nabla u\|_{H^{s}}\|B\|_{H^{s}}^{2}
$$

which implies that

$$
\|B(t)\|_{H^{s}}^{2} \leqq\left\|B_{0}\right\|_{H^{s}}^{2} \exp \left(2 c_{4} \int_{0}^{t}\|\nabla u\|_{H^{s}} \mathrm{~d} \tau\right) ;
$$

and the maximal regularity estimate

$$
\begin{aligned}
\int_{0}^{T}\|u\|_{H^{s+1}} \leqq & C_{\varepsilon} T^{\varepsilon / 2}\left\|u_{0}\right\|_{H^{s-1+\varepsilon}} \\
& +C_{\varepsilon} T^{\varepsilon /(s+\varepsilon)}\left(\int_{0}^{T}\|B\|_{H^{s}}^{2(s+\varepsilon) / s}+c_{5} M_{0}^{\varepsilon / s}\|u\|_{H^{s+\varepsilon}}^{2} \mathrm{~d} \tau\right)^{s /(s+\varepsilon)} .
\end{aligned}
$$

We will now choose $T^{*}$ such that $\|B(t)\|_{H^{s}} \leqq 2\left\|B_{0}\right\|_{H^{s}}$ for all $t \in\left[0, T_{*}\right]$. Set

$$
\begin{aligned}
M_{1} & :=\left\|u_{0}\right\|_{H^{s-1+\varepsilon}}, \\
\text { and } \quad M_{2} & :=2^{2(1+\varepsilon)} c_{2}\left\|B_{0}\right\|_{H^{s}}^{2(1+\varepsilon)}+2^{4} c_{3}\left\|B_{0}\right\|_{H^{s}}^{4}+2 M_{0},
\end{aligned}
$$

and choose $T^{*}$ sufficiently small that

$$
0<\left(1-\frac{c_{1} T\left(M_{1}^{2}+T M_{2}\right)^{1 / \varepsilon}}{\varepsilon}\right)^{-\varepsilon}<2 \text { for all } 0<T<T_{*}
$$

${ }^{1}$ Note that the exponent $2(1+\varepsilon) / \varepsilon$ on the $H^{s-1+\varepsilon}$ norm of $u$ is far from optimal, and can be reduced to some $\gamma$ for $2<\gamma \leqq 4$ by using Lemma 1.1(i) from [4]. However, the proof here is significantly simpler, and still yields a short-time existence result (albeit with a possibly shorter existence time). 
and

$$
\begin{aligned}
C_{\varepsilon} T^{\varepsilon / 2} M_{1} & +C_{\varepsilon} T^{\varepsilon /(s+\varepsilon)}\left(2^{2(s+\varepsilon) / s} T\left\|B_{0}\right\|_{H^{s}}^{2(s+\varepsilon) / s}\right. \\
& \left.+c_{5} M_{0}^{\varepsilon / s}\left[c_{1} T\left[2\left(M_{1}^{2}+T M_{2}\right)\right]^{(1+\varepsilon) / \varepsilon}+T M_{2}\right]\right)^{s /(s+\varepsilon)}<\frac{\log 4}{2 c_{4}}
\end{aligned}
$$

for all $0<T<T_{*}$.

To show that $\|B(t)\|_{H^{s}} \leqq 2\left\|B_{0}\right\|_{H^{s}}$ for $t \in\left[0, T_{*}\right]$, we make the assumption that $t \mapsto\|B(t)\|_{H^{s}}$ is a continuous function that takes the value $\left\|B_{0}\right\|_{H^{s}}$ at time $t=0$. While we have not shown this as part of our formal calculations, it would be true for any member of the family of smooth approximations considered in [6], and the estimates we now obtain would hold uniformly (for this family) for all $t \in\left[0, T^{*}\right]$ for the time $T^{*}$ defined by (3.12) and (3.13).

Set

$$
T=\sup \left\{T_{0} \in\left[0, T^{*}\right]:\|B(t)\|_{H^{s}} \leqq 2\left\|B_{0}\right\|_{H^{s}} \quad \text { for all } \quad t \in\left[0, T_{0}\right]\right\}
$$

and suppose that $T<T^{*}$. Then from (3.1), (3.2) and (3.9) we obtain

$$
\begin{aligned}
\frac{\mathrm{d}}{\mathrm{d} t}\|u\|_{H^{s-1+\varepsilon}}^{2}+\|u\|_{H^{s+\varepsilon}}^{2} \leqq & c_{1}\|u\|_{H^{s-1+\varepsilon}}^{2(1+\varepsilon) / \varepsilon}+2^{2(1+\varepsilon)} c_{2}\left\|B_{0}\right\|_{H^{s}}^{2(1+\varepsilon)} \\
& +2^{4} c_{3}\left\|B_{0}\right\|_{H^{s}}^{4}+2 M_{0} \\
\leqq & c_{1}\|u\|_{H^{s-1+\varepsilon}}^{2(1+\varepsilon) / \varepsilon}+M_{2}
\end{aligned}
$$

for all $t \in[0, T]$. Using standard ODE comparison techniques (see, for example, Theorem 6 in [2], where we take $p=(1+\varepsilon) / \varepsilon)$ we obtain the bound

$$
\begin{aligned}
\|u(t)\|_{H^{s-1+\varepsilon}}^{2} & \leqq\left(M_{1}^{2}+T M_{2}\right)\left(1-\frac{c_{1} T\left(M_{1}^{2}+T M_{2}\right)^{1 / \varepsilon}}{\varepsilon}\right)^{-\varepsilon} \\
& \leqq 2\left(M_{1}^{2}+T M_{2}\right)
\end{aligned}
$$

for all $t \in[0, T]$, by (3.12).

Now, substituting (3.15) into (3.14) and integrating between times 0 and $T$ yields

$$
\int_{0}^{T}\|u(t)\|_{H^{s+\varepsilon}}^{2} \mathrm{~d} t \leqq c_{1} T\left[2\left(M_{1}^{2}+T M_{2}\right)\right]^{(1+\varepsilon) / \varepsilon}+T M_{2} .
$$

Substituting (3.16) and $\|B(t)\|_{H^{s}} \leqq 2\left\|B_{0}\right\|_{H^{s}}$ into (3.11), we obtain

$$
\begin{aligned}
\int_{0}^{T}\|u(t)\|_{H^{s+1}} \mathrm{~d} \tau \leqq & C_{\varepsilon} T^{\varepsilon / 2} M_{1}+C_{\varepsilon} T^{\varepsilon /(s+\varepsilon)}\left(2^{2(s+\varepsilon) / s} T\left\|B_{0}\right\|_{H^{s}}^{2(s+\varepsilon) / s}\right. \\
& \left.+c_{5} M_{0}^{\varepsilon / s}\left[c_{1} T\left[2\left(M_{1}^{2}+T M_{2}\right)\right]^{(1+\varepsilon) / \varepsilon}+T M_{2}\right]\right)^{s /(s+\varepsilon)} \\
& <\frac{\log 4}{2 c_{4}}
\end{aligned}
$$

using (3.13). 
Substituting this into (3.10) ensures that $\|B(t)\|_{H^{s}}<2\left\|B_{0}\right\|_{H^{s}}$ for all $t \in$ $[0, T]$, contradicting the maximality of $T$. It follows that $T=T^{*}$ and hence

$$
\|B(t)\|_{H^{s}} \leqq 2\left\|B_{0}\right\|_{H^{s}} \quad \text { for all } t \in\left[0, T^{*}\right] .
$$

The result now follows from (3.15), (3.16), and (3.17).

\section{Conclusion}

In the scale of Sobolev spaces we suspect that the result that we have proved here is optimal. Bourgain and Li [3] showed that the Euler equations on $\mathbb{R}^{d}$ are ill posed in $H^{1+d / 2}$ for $n=2,3$, and we have shown via an explicit example that for the heat equation we cannot gain the time integrability of two additional derivatives that is required in our local existence argument. It would be interesting to find a simpler model problem in which it is possible to demonstrate the failure of local existence for $B_{0} \in H^{s}$ and $u_{0} \in H^{s-1}$.

Conflict of interest The authors declare that they have no conflict of interest.

Open Access This article is distributed under the terms of the Creative Commons Attribution 4.0 International License (http://creativecommons.org/licenses/by/4.0/), which permits unrestricted use, distribution, and reproduction in any medium, provided you give appropriate credit to the original author(s) and the source, provide a link to the Creative Commons license, and indicate if changes were made.

\section{References}

1. Benedek, A., Calderón, A.-P., Panzone, R.: Convolution operators on Banach space valued functions. Proc. Nat. Acad. Sci. 48, 356-365 (1962)

2. Blömker, D., Nolde, C., Robinson, J.C.: Rigorous numerical verification of uniqueness and smoothness in a surface growth model. J. Math. Anal. Appl. 429(1), 311-325 (2015)

3. Bourgain, J., Li, D.: Strong ill-posedness of the incompressible Euler equation in borderline Sobolev spaces. Invent. Math. 201(1), 97-157 (2015)

4. Chemin, J.-Y.: Remarques sur l'existence globale pour le système de Navier-Stokes incompressible. SIAM J. Math. Anal. 23(1), 20-28 (1992)

5. Chemin, J.-Y., McCormick, D.S., Robinson, J.C., Rodrigo, J.L.: Local existence for the non-resistive MHD equations in Besov spaces. Adv. Math. 286, 1-31 (2016)

6. Fefferman, C.L., McCormick, D.S., Robinson, J.C., Rodrigo, J.L.: Higher order commutator estimates and local existence for the non-resistive MHD equations and related models. J. Funct. Anal. 267(4), 1035-1056 (2014)

7. JiU, Q., Niu, D.: Mathematical results related to a two-dimensional magnetohydrodynamic equations. Acta Math. Sci. Ser. B Engl. Ed. 26(4), 744-756 (2006)

8. Krylov, N.V.: The heat equation in $L_{q}\left((0, T), L_{p}\right)$-spaces with weights. SIAM J. Math. Anal. 32(5), 1117-1141 (2001)

9. Ladyzhenskaja, O. A., Solonnikov, V.A., Ural'ceva, N.N.: Linear and quasilinear equations of parabolic type. Translated from the Russian by S. Smith. Translations of Mathematical Monographs, Vol. 23, American Mathematical Society, Providence, 1968

10. Lin, F., Xu, L., Zhang, P.: Global small solutions to 2D incompresible MHD system. J. Differ. Equ. 259, 5440-5485 (2015) 
11. Minlin, S.G.: Fourier integrals and multiple singular integrals. Vestnik Leningrad. Univ. Ser. Mat. Meh. Astr. 12(7), 143-155 (1957)

12. Ren, X., Wu, J., XiAng, Z., Zhang, Z.: Global existence and decay of smooth solution for the 2D MHD equations without magnetic diffusion. J. Funct. Anal. 267, 503-541 (2014)

\author{
Charles L. FefFerman \\ Department of Mathematics, \\ Princeton University, \\ Fine Hall, Washington Road, \\ Princeton, NJ 08544, \\ USA.
}

e-mail: cf@math.princeton.edu and

DAvid S. MCCORMICK

School of Mathematical and Physical Sciences,

University of Sussex, Pevensey 2,

Brighton BN1 9QH,

UK.

e-mail: d.s.mccormick@ sussex.ac.uk

and

James C. Robinson \& Jose L. Rodrigo

Mathematics Institute,

University of Warwick, Coventry CV4 7AL, UK.

e-mail: j.c.robinson@warwick.ac.uk e-mail: j.rodrigo@warwick.ac.uk

(Received February 5, 2016 / Accepted August 18, 2016)

Published online September 1, 2016 - () The Author(s) (2016)

This article is published with open access at Springerlink.com 